\title{
A Fuzzy Logic Framework for Evaluating the Security Features of Banknotes
}

\author{
Nagy Ramadan Darwish \\ Department of Information Systems \&Technology, \\ Institute of Statistical Studies and Research, Cairo \\ University
}

\author{
Ashraf M. EL Nour \\ Department of Information Systems \&Technology \\ Institute of Statistical Studies and Research, Cairo \\ University
}

\begin{abstract}
Watermark, security thread, hologram and Intaglio printing are important features for securing banknotes can be realized by the user senses. The goal of these features is to provide sufficient quality features that can help the public to determine whether or not their banknote is genuine. Recently, due to the development in computer software, laser printers and scanners, counterfeiting has become a vital issue. This research aims to generate evaluation security features, for banknotes model in order to enhance the quality. A Quantitative approach for evaluation the quality of security features banknotes, where the data gathered via a questionnaire using the five-point Likert scale that uses the values: poor, fair, good, very good, and excellent. The proposed framework depends on the utilization of fuzzy logic for classification banknotes genuine or counterfeit. However, fuzzy logic provides useful techniques for dealing with decisions in such environments which contain imprecise and vague values.
\end{abstract}

\section{General Terms}

Software Engineering, Evaluation Approaches, Banknotes Design.

\section{Keywords}

Banknotes Security Features, Counterfeiting, Likert Scale, Fuzzy Logic, Software Development.

\section{INTRODUCTION}

The quality of banknotes in circulation is an important task to central bank of countries in order to the replacement of banknotes that are worn or damaged and elimination counterfeiting. Damaged or tatty banknotes late payment transactions and can make some security features hard or impossible to use. This effect to both manual and automated authentication. When it becomes more difficult to determine whether banknotes are genuine or not confidence will weaken. Worn banknotes may reason Automated Teller Machines (ATMs) to jam, growing the need for maintenance, and vending machines might reject banknotes that are too worn to be authenticated or handled mechanically [1].

Nowadays, the countries facing the growing and the challenging difficulties of counterfeiting of its banknotes. A challenge has been emerged to stop counterfeiting of currency the key point is to improve the quality of security features. Security features can be classified into three groups, (1) substrate features like watermark, security fiber's, see through windows. (2) Ink features for instance color shifting inks, UV glowing ink, infrared ink, etc.(3) And printing features as Guilloches, Intaglio printing, micro-printing, that are only known by expert forensics examiners [2], but due to advancement of the printing media technology, it has become an easy task to counterfeit paper banknotes. The increase of counterfeit banknotes causes damage to banks, traders, and individuals involved in financial transactions. Hence the fake banknotes' detection is an emerging issue for a country to protect its economy as well as people's faith on currency [3].

The general requirements of security features of banknotes include the following [4]:

- Acceptable to the public.

- $\quad$ Easy to find and understand.

- Quick to identify without any possibilities of errors.

- Machine readable.

- $\quad$ Easy to produce at low cost.

- Extremely difficult to duplicate.

- Durable (remains visible after considerable wear).

- Ability to integrate with each other and the design to produce additional more complex security features.

- Nontoxic and non-hazardous.

Software development is a mentally complicated task. Therefore, different software development methodologies and quality assurance methods are used in order to attain high quality, reliable, and bug free software. In recent year's evaluation the quality security features banknotes to detect counterfeits and the replacement of banknotes that are worn or damaged have gained much attention in the field of software engineering [1]. One of the important goals of this exercise is to enable the central bank and experts to examine the quantitative feedback of the public, which are computed and analyzed that provide valuable insights and helps the experts take note of their strengths and weaknesses of security features banknotes [5].

In this paper, the researchers propose a conceptual framework for the public evaluation the security features of banknotes, the feedback analysis that provides a starting point for community of stakeholders to consider how qualitative and quantitative feedback can help in making informed decisions with respect to quality and curriculum improvements. The proposed framework depends on Likert scales and utilization of fuzzy logic for classification banknotes genuine or counterfeit.

The rest of this paper is divided into nine sections. Section 2 background introduces an overview on Likert scales that includes main characteristics, and presents a brief introduction of fuzzy logic, and finally different techniques to detect counterfeiting. Section 3 introduces a literature that includes some important papers and researches in quality banknotes, 
Section 4 introduces the proposed framework. Section 5 Experimental Design. Section 6 introduces the Experimental Results and Discussion Section 7 Validation of the proposed framework. Section 8 introduces the conclusion of the paper. Section 9 introduces the ideas that are expected to be focused in the future.

\section{BACKGROUND}

This section provides an overview about the main concept related to the research topic. It consists of two parts. In the first part, a questionnaire using the five-point Likert Scale which are used as a standard scale to measure feedback of the user about security features of banknotes is presented. In the second part, the basic concept of fuzzy logic is discussed.

\subsection{Likert Scale}

Likert scales have been commonly used to measure observable attributes in various social science measurement areas. It is a psychometric scale commonly involved in research that employs questionnaires. It is the most used approach to scaling response in survey research. Likert scales are a non-comparative scaling technique and are one dimensional in nature. When responding to a Likert questionnaire item respondent specify their level of agreement or disagreement on a symmetric agree- disagree scale for a series of statements. It is an easy way to evaluate qualitative, linguistic statements is to convert them into numerical values with simple ranking. The advantage of this ranking technique is its simplicity, the numerical measurement results can be directly used for statistical inference and researchers can collect and analyze a large quantity of data with less time and effort.

The Likert scale is commonly used as a standard psychometric scale to measure feedback of the public about banknotes. This measurement scale has a procedure that facilitates survey construction, and analysis. A Quantitative approach for evaluating the security features banknotes where the data gathered via a questionnaire using the five-point Likert that uses the values: poor, fair, good, very good, and excellent. [6].

\subsection{Fuzzy Logic}

Fuzzy Logic (FL) is a methodology to solve problems which are too complex to be understood quantitatively. It is based on "degrees of truth" rather than the traditional Boolean logic "true or false" on which the modern computer is based. It allows us to provide the values of elements in human such as language values (such as high, low and moderate) with a high accuracy in the calculation process. The Fuzzy Logic System deals with fuzzy parameters, which address imprecision and uncertainties, by mapping out the path of a given input to an output using the computing framework called the Fuzzy Inference System (FIS).

A fuzzy Inference system is a knowledge based or rule based system. FIS consists of four components, they are: Fuzzifier, Fuzzy Rule Base, Fuzzy Inference Engine, and Defuzzification. The fuzzifier converts the crisp input into a fuzzy set that is a non- traditional type of sets which allows an element to have a partial degree of membership. The membership refers to the degree of inclusion to specifically set. There are many membership functions but in this research we will use the triangle membership function (see Figure 1)

A fuzzy inference engine is a collection of IF -THEN rules stored in the fuzzy rule base are known as inference engine. Defuzzification is the process which refers to the transform of the fuzzy output into crisp output. The most common tools that are used for fuzzy systems is MATLAB which used for defining the input, output, inference rules, and the shape of membership function for the fuzzy system. Fuzzy logic provides the concept of fuzzy sets to handle vague and inaccurate data [7].
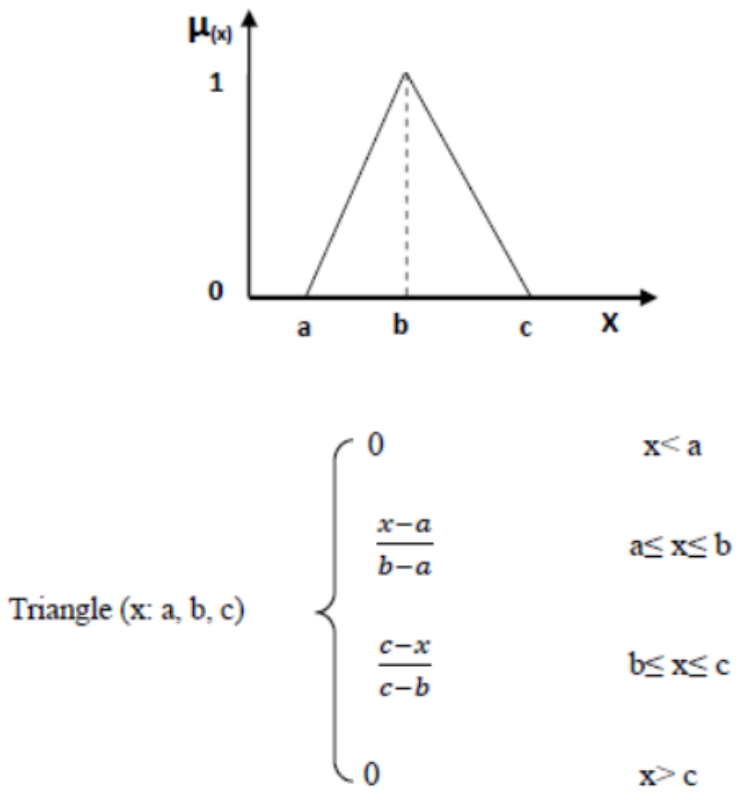

Fig 1: Triangular membership function.

\subsection{Techniques to detect counterfeiting}

The most techniques used in the production of banknote printing involve security paper, security ink, lithography Security, intaglio printing, micro-printing, machine reading, durability, and design [17].

\subsubsection{Security Paper}

Security paper demand many Security features for instance; (watermark; multi-tone watermarks; ultraviolet-dull; Security threads and fibber's). The watermark is the major and most secure feature because the public easily recognizes it. Furthermore, multi-tone watermarks offers protection against scanners, copiers, chemical mechanism, and reproduction trial. Security paper must be ultraviolet-dull (UV-dull), in which that paper is free from optical brightening agents. This UV-dull paper improves security for two reasons. One, "It allows for the use of fluorescent inks as a security feature, and two, by not fluorescing under UV light as would counterfeits produced on ordinary, brightened coloured paper".

Security threads and fibres are else component of security that involves the substrate similar to a watermark. Security fibres offer visibly different colour fibres to the documents. That is easily detectable by the naked eye. Also, security fibres can be almost invisible colours that fluoresce when illuminated with UV light, making authentication easy. Security fibres are often used in addition to a watermark and also provide protection against colour photo-copying.

\subsubsection{Security Ink}

There are two major types of ink associated with security printing. The first is an anti-duplication ink, and the second is an anti-alteration ink. "Anti-duplication inks are designed to block unauthorized copying or duplication of documents". "Anti-alteration inks provide overt indications of tampering, such as discoloration upon exposure to water or chemicals or 
destruction to the background". Ultraviolet Inks (UV) "cannot be seen with the human eye under normal lighting conditions", these inks requires light from UV radiation. This ink offers a covert security feature by not being detectable without the proper short-wave, or long-wave UV illumination

\subsubsection{Security lithography}

Security lithography include creating the images of using a line structure formation and altering the colours along this line. Simulated 3-D effects are achieved with this type of lithography and are fundamental component in preventing an easy replication from a counterfeiter. Using lithography, it is possible to print a smoothly changing colour along a single printed line.

\subsubsection{Intaglio Printing}

Intaglio printing is the essential printing process is used because its capacity to produce extremely fine detail that remains legible under repeated handling and is difficult to counterfeit. However, intaglio security printing involves, "Thick paste inks with hand-engraved plates using high printing pressures" .Moreover, intaglio security printing "has the characteristic variation in depth of image and this variation in tonal range".

\subsubsection{Micro-printing}

Micro-printing is a popular security printing technique that refers to printing microscopic letters that are, "too small to see without magnification". These characters look like a very thin line, however microprints contain much more legible information. Micro-printing is hard to counterfeit because it is practically impossible to reproduce the print accurately with only the use of photocopiers.

\subsubsection{Machine Reading}

Machine reading is utilize of a machine to authenticate the legitimacy of a document. There are two major features of machine reading. The first types are features are known as teller-assisted features, which help inspectors to authenticate documents. The second types are automated features that are used to activate the acceptance of a document. Fluorescence lighting would be an example of automated features, and a machine sorting system would be an example of the latter.

\subsubsection{Durability}

Durability is appropriate to every type of security document. When making a security document such as a banknote, there should always be thought as to how this document will be handled during its lifetime. The handling environment of a security document plays a large part in determining what security features can be incorporated and what type of crime. Banknote is a distinctive type of document because they are first supplied by a central bank and then circulated through the economic waves of society. There is no specific environment that the banknote must be contained within. Hence cultural and social climate dictates whether the document is in a wallet, billfold, pocket, or just held next to the skin. Exposure to hazards is high as the document is handled under all conditions, from banks, offices, ships, markets, and bars. Under these conditions, it must represent its value and not change colour or nature. Banknotes must be durable to ensure that the security devices used in their production last for the entire life of the banknote.

\subsubsection{Design}

One of the most important design parameters for optimal value recognition by the public is the main, dominant colour of the note [18].Security designers must combine fine artists with the skills of a forensic scientist. If security designers do not make this combination of design skills and science skills, then they will be significantly less secure against counterfeiters and forgers. An effective design for a secure banknote will give the impression of authority and security of the country they represent. The high quality of the banknote design in circulation helps the public detect counterfeits. Furthermore, banknote must be acceptable to retailers and be capable of being used in banknote equipment, such as automated teller machines (ATMs) and vending machines.

\section{LITERATURE REVIEW}

There are many papers and researches in the quality of banknotes. The following are some examples of these literatures:

Trond E. et al, this research aims to explain why the quality of banknotes in circulation is important and discusses the challenges regarding the quality of banknotes in circulation, and the possibility to monitor and influence quality using a concept developed and implemented. Finally, explain the relationship between quality and efficiency [1].

Harald D. et al, this research presents two computer-based models for the simulation of banknote cash cycles. The first model simulates a cash cycle using a theoretical approach based on key figures and models banknote fitness as a onedimensional profile of fitness levels. The model identifies: (I) the frequency with which banknotes are returned to the central bank; (ii) the fitness threshold used in automated note processing at the central bank; and (iii) the note lifetime as the main drivers of banknote quality in circulation as well as central bank cash cycle costs. Production variations in new banknotes, the fitness threshold applied by commercial cash handlers and the accuracy of the fitness sensors used in the sorting process have been found to have a lower but nontrivial impact. The second model simulates banknotes in circulation as single entities and is oriented towards modelling country-specific cash cycles using available single-note data. The model is constructed using data collected by monitoring banknotes in circulation over the duration of a "circulation trial" carried out in three euro area countries [8].

Arianna C. et al, this research aims to keep the quality of banknotes in circulation, high to ensure that they meet the needs of the public and to make it more difficult for counterfeits to be passed or remain in circulation. Discusses the quality of banknotes in Australia and Reserve Bank initiatives that have improved the quality of banknotes in recent years [9].

Frank V. et al, introduced contribution depend on Counterfeit prevention is a major task for central banks, which often maintain that it is easier for the public to detect counterfeits if the quality of the banknotes in circulation is high. This research provide evidence for the link between the quality of banknotes and counterfeit detection with respect to euro banknotes. In addition, refinement the methodology of the few existing studies on counterfeit detection by investigating detection rates and the incidence of false alarms simultaneously [10].

Alexandra R., this research presents proposes an alternative way of studying banknote life by estimating survival models, which are commonly used in studying life expectancies in medicine or the duration of events in economics. These models can produce estimates of central tendency that are much less volatile over time and provide information on the probability that banknotes survive over time. The models' 
predictions of banknote survival are intuitive and the results are consistent with samples of circulating and unfit banknotes [11].

Jan M. et al, introduced a machine learning method is proposed for banknote soiling determination, and apply proven techniques from computer vision to come up with a robust and effective method for automatic sorting of banknotes. The proposed method is evaluated with respect to various invariance classes. The method shows excellent performance on a large validation set of over 8,000 banknotes from the Euro system, while being learned on only 300 banknotes per denomination [12].

Seung Y. et al, introduced Fitness classification is a technique to assess the quality of banknotes in order to determine whether they are usable. Banknote classification techniques are useful in preventing problems that arise from the circulation of substandard banknotes. Fitness classification continues to be carried out by humans, and this can cause the problem of varying fitness classifications for the same bill by different evaluators, and requires a lot of time. To address these problems, this study proposes a fuzzy system- based method that can reduce the processing time needed for fitness classification, and can determine the fitness of banknotes through an objective, systematic method rather than subjective judgments [13].

Michael A. et al, introduced several banknote quality initiatives have been introduced in Australia over the past decade. The most recent of these note quality reward scheme was explicitly designed to improve the quality of banknotes in circulation, to improve banknote fitness sorting behaviour and encourage investment in banknote fitness sorting technologies. Despite having only been introduced in late 2006 , there is already considerable evidence to suggest that these objectives are being realised. Substantial investment by the private sector in banknote processing machines has increased their ability to out sort poor quality banknotes which, in turn, has led to a demonstrable improvement in banknote quality across all denominations [14].

Osamu M. et al, introduced the value of a banknote is dependent on people's subjective trust in the banknote, and the resistance against counterfeiting is a key factor of people's confidence in the banknote. An experiment was conducted to investigate the relationships between the awareness to security features on banknotes and the perceived resistance against counterfeiting in those bank notes. It was found that the more security features subjects found by themselves on a banknote, the more resistant they perceived the banknote, which suggests that people's awareness to security features affects their confidence in the bank note. The perceived resistance was irrelevant to the number of public security features disclosed by the central banks, but was relevant to the familiarity to the note, which suggests the importance of practical experience with banknotes rather than the knowledge about them only. These findings can give a quantitative ground to the evaluation of the design of security features on banknotes [15].

Marius p.et al, introduced Portraits have been thought to be one of the most important security features on banknotes for a long time. Portraits are often printed as the main feature of banknotes at a very high resolution in intaglio press. This research investigate on how important portraits actually are and what factors were contributing to the perceived genuineness of portraits on banknotes with a psychometric experiment. Banknotes were presented to subjects, enclosed in envelopes to cover the area other than the portrait, and the genuineness of those banknotes were rated. The factors affecting the rating were asked to the subjects at the same time. It was suggested that natural wear and tear strengthen the perceived genuineness of testing banknotes. Even though the inspection of the banknotes was restricted to on and around the portrait, the importance of the portrait was not high compared to other features, and a significant fraction of the subjects answered that they paid no attention to any part of the face, which requires reflection and reconsideration of the use of human portraits as a security feature [16]. The purpose of this study is to know whether Watermark, security thread, hologram and Intaglio printing are actually an important as a security feature banknotes and how they are contributing to the perceived genuineness of banknotes.

\section{THE PROPOSED FRAMEWORK}

This paper presents a Quantitative approach for user evaluation the security features (Watermark, security thread, hologram, and Intaglio printing) of banknotes. We are going to propose an adapted questionnaire then describe the data analysis should be used to get results of the questionnaire that evaluate the security features of banknotes. A questionnaire using the five-point Likert scale that uses the values: poor, fair, good, very good, and excellent. The framework will help in quantifying requirements from simple yes/no to reach more comprehensive feedback [19]. Figure 2 shows that the flowchart of the proposed framework starts with inputs one thousand Egyptian currency notes $(100,50,20,10)$ pounds, contain genuine, Counterfeit and worn .We ask a random sample of the public people, one thousand person to provide qualitative responses on their perceptions about the security features of banknotes using sight, touch and sound. The long list of qualitative feedback is given about the security features of banknotes (Watermark F1, security thread F2, hologram F3 and Intaglio printing F4). Capturing and analysing the qualitative feedback data can provide valuable insights on the strengths and weaknesses of security features banknotes.

- IF F1, F2, F3, and F4 are poor indicate Counterfeit.

- $\quad$ IF F1, F2, F3, and F4 are fair indicate genuine.

- $\quad$ IFF1, F2, F3, and F4 are good indicate genuine.

- IF F1, F2, F3, and F4 are very good indicate genuine.

- $\quad$ IFF1, F2, F3, and F4 are Excellent indicate genuine.

- IF F1, F2, F3 are poor and F4 are fair indicate Counterfeit.

- $\quad$ IF F1 is poor and F2, F3, F4 are fair indicate worn.

- IFF1, F2 are poor and F3, F4 are fair indicate worn.

The proposed framework depends on the utilization of fuzzy logic for classification currency genuine, counterfeit and worn. In the first step, the inputs are fuzzified, to obtain fuzzy sets using the triangular membership function. The second step, the crisp values from first step are converted into linguistic variables. It means that each crisp input value is transformed into grades of membership for linguistic terms of fuzzy sets. In the training phase, the features vector is utilized to build the membership function for each feature according to the minimum and maximum values of that feature. These membership functions are used later in the testing phase to fuzzify the features. The parameters for the MFs are obtained by training the system with the genuine currency notes of the 
user. During training, the parameters are tuned iteratively in order to minimize the mean square error of the output. The third step called fuzzy inference or approximate reasoning. They are used in a fuzzy rule to determine the rule outcome from the given rule input information.

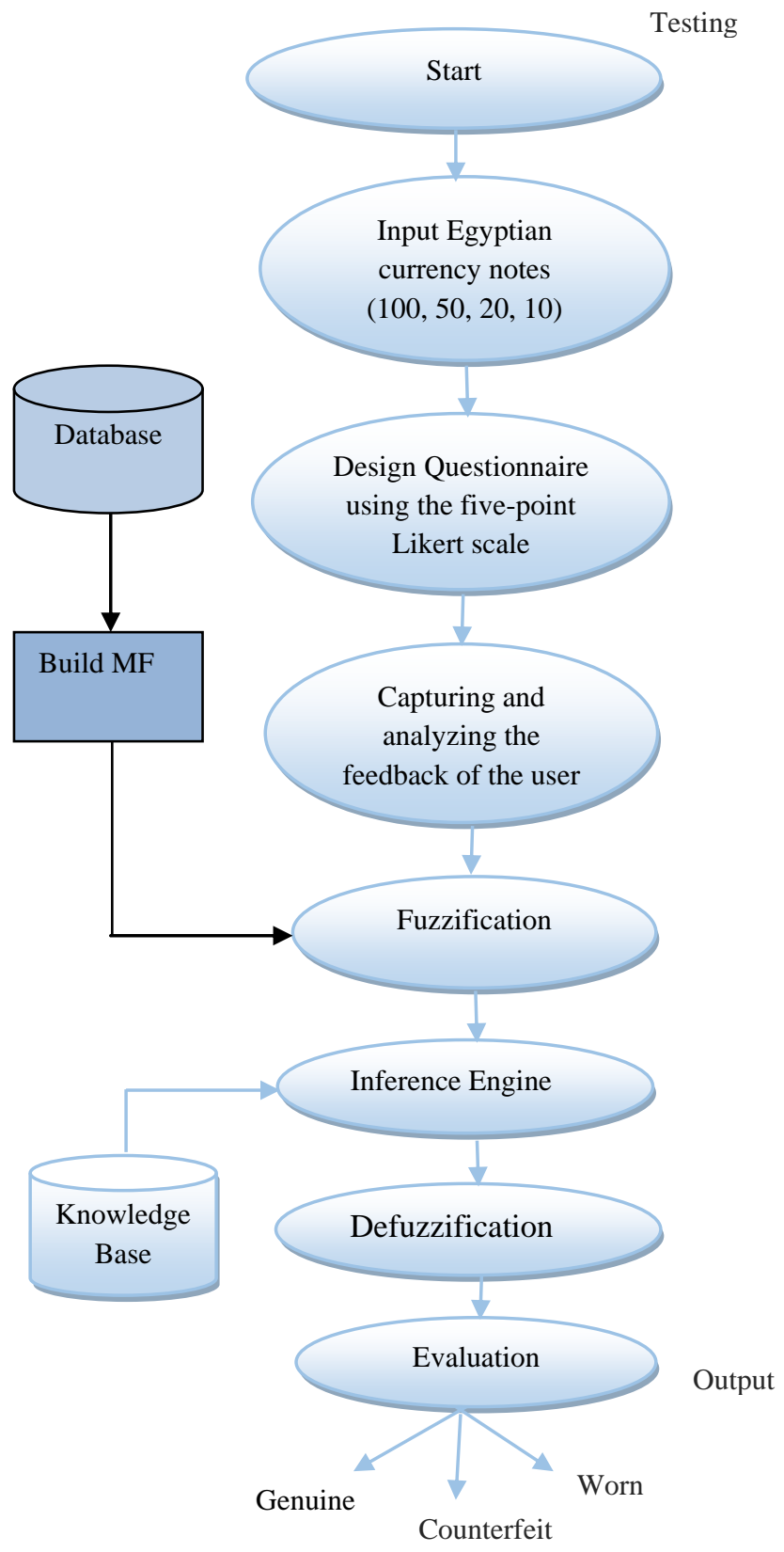

Fig 2: Proposed Framework Flowchart

Fuzzy rules represent modelling knowledge / experience. When specific information is assigned to input variables in the rule antecedent, fuzzy inference is needed, to calculate the outcome for output variables in the rule consequence. Fuzzy reasoning (FR) which is formulated by group of fuzzy IFTHEN rules, presents a degree of presence or absence of an association or interaction between the elements of two or more sets. The most popular fuzzy rules are Mamdani fuzzy rules. Herein, three fuzzy variables, including 'very small', small and 'large' are used to describe the feature variations. In the proposed system, reasoning is carried out through the following rules:

\section{Rule 1}

IF (f1 is small) and (f2 is small) and ( $\mathrm{f} 3$ is small) and (f4 is small) THEN (output is very small) $=$ Counterfeit.

Rule 2

IF ( $\mathrm{f} 1$ is large) and (f2 is large) and ( $\mathrm{f} 3$ is small) and ( $\mathrm{f} 4$ is small) THEN (output is small) $=$ Worn .

\section{Rule 3}

IF ( $\mathrm{f} 1$ is small) and (f2 is large) and (f3 is small) and ( $\mathrm{f} 4$ is small) THEN (output is very small) $=$ Counterfeit.

Rule 4

IF ( $\mathrm{f} 1$ is small) and ( $\mathrm{f} 2$ is small) and ( $\mathrm{f} 3$ is large) and ( $\mathrm{f} 4$ is small) THEN (output is very small)= Counterfeit.

\section{Rule 5}

IF ( $\mathrm{f} 1$ is small) and (f2 is small) and ( $\mathrm{f} 3$ is small) and ( $\mathrm{f} 4$ is large) THEN (output is very small) $=$ Counterfeit

Rule 6

IF ( $\mathrm{f} 1$ is small) and (f2 is small) and ( $\mathrm{f} 3$ is large) and ( $\mathrm{f} 4$ is large) THEN (output is small) $=$ Worn

Rule 7

IF ( $\mathrm{f} 1$ is small) and (f2 is large) and ( $\mathrm{f3}$ is small) and ( $\mathrm{f} 4$ is large) THEN (output is small) $=$ Worn .

Rule 8

IF ( $\mathrm{f} 1$ is large and ( $\mathrm{f} 2$ is small) and ( $\mathrm{f} 3$ is large) and ( $\mathrm{f} 4$ is small) THEN (output is small) $=$ Worn .

Rule 9

IF ( $\mathrm{f} 1$ is large) and (f2 is large) and (f3 is small) and (f4 is small) THEN (output is small)= Worn.

Rule 10

IF ( $\mathrm{f} 1$ is large) and (f2 is large) and (f3 is large) and (f4 is small) THEN (output is large) $=$ genuine .

Rule 11

IF ( $\mathrm{f} 1$ is large) and (f2 is large) and ( $\mathrm{f} 3$ is small) and ( $\mathrm{f} 4$ is large) THEN (output is large) $=$ genuine

Rule 12

IF (f1 is large) and (f2 is small) and (f3 is large) and (f4 is large) THEN (output is large) = genuine

Rule 13

IF (f1 is small) and ( $\mathrm{f} 2$ is large) and ( $\mathrm{f} 3$ is large) and (f4 is large) THEN (output is large) $=$ genuine

Rule 14

IF ( $\mathrm{f} 1$ is large) and (f2 is small) and (f3 is large) and ( $\mathrm{f} 4$ is large) THEN (output is large) $=$ genuine .

Rule 15

IF ( $\mathrm{f} 1$ is large) and (f2 is large) and (f3 is small) and (f4 is large) THEN (output is large $)=$ genuine .

Rule 16

IF ( $\mathrm{f} 1$ is large) and (f2 is large) and (f3 is large) and ( $\mathrm{f} 4$ is large) THEN (output is large) $=$ genuine . 
These rules are built based on the experiences of the expert of a forgery and counterfeiting, department of the forgery and counterfeiting researches, Forensic Medicine - Ministry of Justice. The sixteen rules altogether deal with the weight assignments impliedly in the same way as what humans experience thinking. The fuzzy inference processes all of the cases in a parallel manner, which makes the decision more reasonable. The outputs of fuzzy values are then defuzzified to generate a crisp value for the variable. There are several defuzzification methods. Each provides a means to choose a single output based on the implied fuzzy sets. Commonly used defuzzifying methods are:

- The mean of maximum method.

- The maximizing decision

- The centres of gravity method.

In this paper, the centres of gravity method is used as a defuzzification strategy [20]. In this case, the fuzzy module acts as a fusing tool to merge different features that is used as a component with the rest of the fuzzy logic components to form a classifier. Figure 3 and Figure 4 shows the Membership functions for input and output.

V.S.

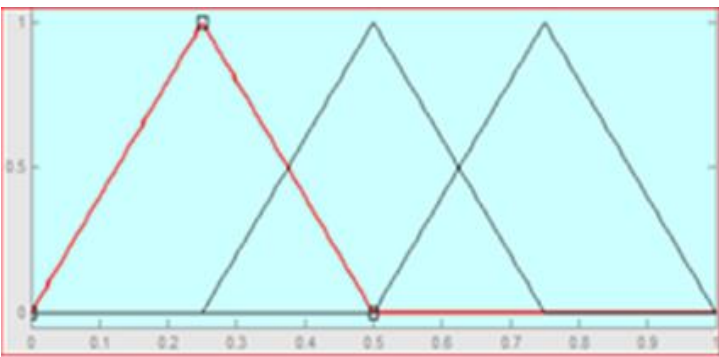

Fig 3: Membership functions for input.

C.

W.

G.

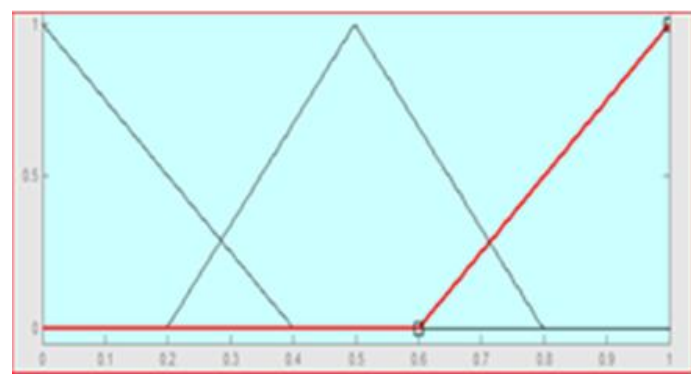

Fig 4: Membership functions for output.

If the output of fuzzy classifier (defuzzification) $\left(\zeta_{2}\right) \geq 0.75$ then the banknotes is genuine (G.), else banknotes is counterfeit(C.).

If the output of fuzzy classifier (defuzzification) $\left(\zeta_{2}\right)=\mathbf{O} .5$ then the banknotes is worn (W.).

\section{EXPERIMENTAL DESIGN}

In order to test the efficiency and validity of the proposed system, the system by MATLAB language was implemented.

The Proposed framework in a modular fashion was built and

it was implemented and tested using a DELL PC machine which had the following features: Intel (R) Core (TM) i5 2450M CPU @ 2.50GHz, and 4.00GB of RAM, 64-bit
Windows 8 Pro. When a respondent examines a banknote in the test set, four outcomes are possible. If the respondent examines a counterfeit banknote, he can either:

- correctly classify the banknote as counterfeit ('hit'), or

- incorrectly classify the banknote as genuine ('miss').

If the respondent examines a genuine banknote, he can either:

- correctly classify the banknote as genuine ('correct reject') or

- $\quad$ incorrectly classify the banknote as counterfeit ('false alarm').

From the number of hits, one can calculate the so-called 'hit rate' (or 'detection rate'), which is defined as the number of hits divided by the total number counterfeits in the test set. Dividing the number of false alarms by the total number of genuine banknotes gives the 'false alarm rate'. Hit rate and false alarm rate jointly quantify the performance of a respondent in the experiment.

\section{EXPERIMENTAL RESULTS AND DISCUSSION}

In this work, one thousand Egyptian currency notes (100, 50, $20,10)$ pounds used in the training phase, and one thousand other was used for testing purposes. one thousand person to provide qualitative responses on their perceptions about the security features of banknotes using sight, touch and sound. See Table 1 for an overview of database for training phase.

Table 1: Database for Training Phase.

\begin{tabular}{|l|l|l|l|l|}
\hline $\begin{array}{l}\text { Type of currency } \\
\text { notes }\end{array}$ & $\begin{array}{l}100 \\
\text { E.P. }\end{array}$ & $\begin{array}{l}50 \\
\text { E.P. }\end{array}$ & $\begin{array}{l}20 \\
\text { E.P. }\end{array}$ & $\begin{array}{l}10 \\
\text { E.P. }\end{array}$ \\
\hline $\begin{array}{l}\text { No. of Genuine } \\
\text { Clean }\end{array}$ & 50 & 50 & 50 & 50 \\
\hline $\begin{array}{l}\text { No. of Genuine } \\
\text { Less Clean }\end{array}$ & 50 & 50 & 50 & 50 \\
\hline $\begin{array}{l}\text { No. of Worn } \\
\text { Clean }\end{array}$ & 50 & 50 & 50 & 50 \\
\hline $\begin{array}{l}\text { No. of Worn } \\
\text { Less Clean }\end{array}$ & 50 & 50 & 50 & 50 \\
\hline $\begin{array}{l}\text { No. of Counterfeit } \\
\text { Clean }\end{array}$ & 25 & 25 & 25 & 25 \\
\hline $\begin{array}{l}\text { No. of Counterfeit } \\
\text { Less Clean }\end{array}$ & 25 & 25 & 25 & 25 \\
\hline
\end{tabular}

Figures 5, 6 and 7 shows the Histogram of category rating in 5-level Likert scale, for (100 pounds) genuine, counterfeiting and worn Egyptian currency notes. 


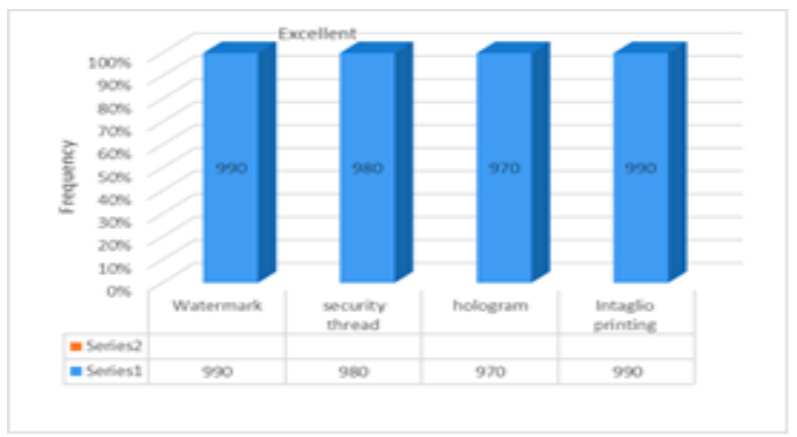

Figure 5: Histogram of category rating in 5-level Likert Scale, for (100 Pounds) genuine Egyptian currency notes.

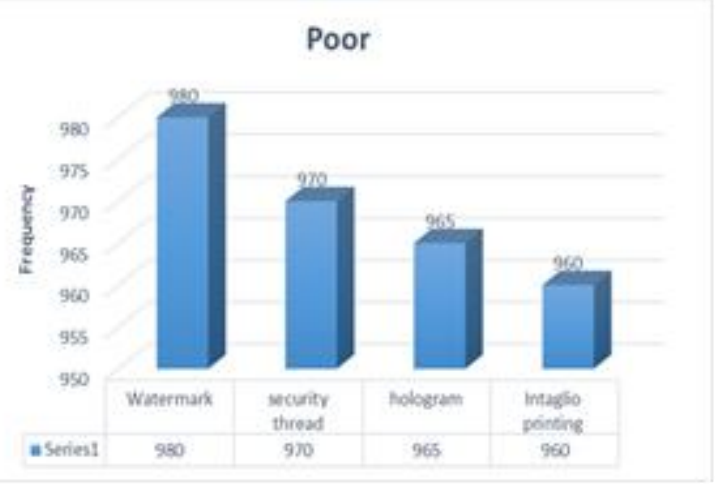

Figure 6: Histogram of category rating in 5-level Likert Scale, for (100 pounds) counterfeiting Egyptian Currency notes.

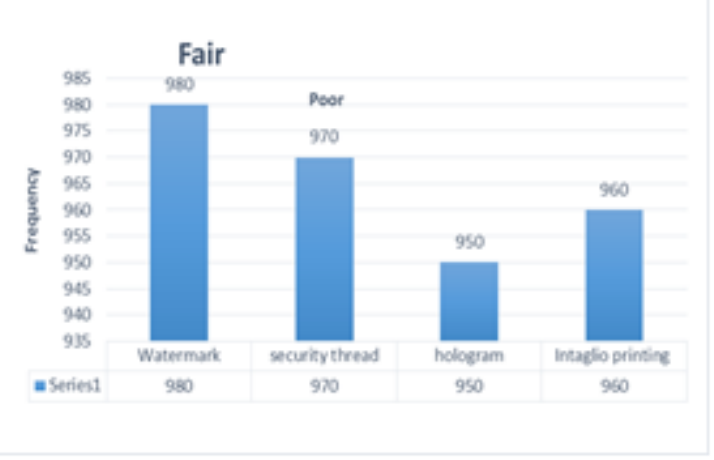

Figure 7: Histogram of category rating in 5-level Likert Scale, for (100 pounds) worn Egyptian currency notes.

Table 2 show the Distribution of the number of security features checked and average hit rate.

\begin{tabular}{|l|l|}
\hline $\begin{array}{l}\text { Number of security } \\
\text { features checked }\end{array}$ & Average hit rate \\
\hline 1 & $75 \%$ \\
\hline 2 & $80 \%$ \\
\hline 3 & $87 \%$ \\
\hline 4 & $96 \%$ \\
\hline
\end{tabular}

Table 3 show the Distribution of the number of security features checked and average False alarm Rate.

\begin{tabular}{|l|l|}
\hline $\begin{array}{l}\text { Number of security } \\
\text { features checked }\end{array}$ & Average False alarm Rate \\
\hline 1 & $20 \%$ \\
\hline 2 & $12 \%$ \\
\hline 3 & $5 \%$ \\
\hline 4 & $2 \%$ \\
\hline
\end{tabular}

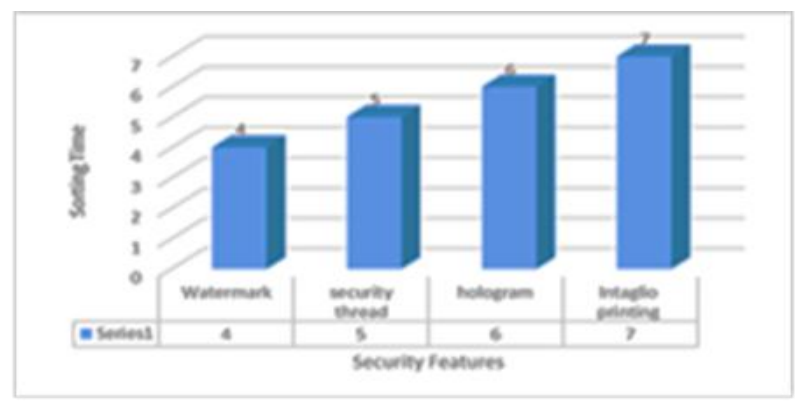

Figure 8 shows the average sorting time in seconds to detect genuine clean Egyptian currency notes $(100,50,20$, 10.) pounds for public people.

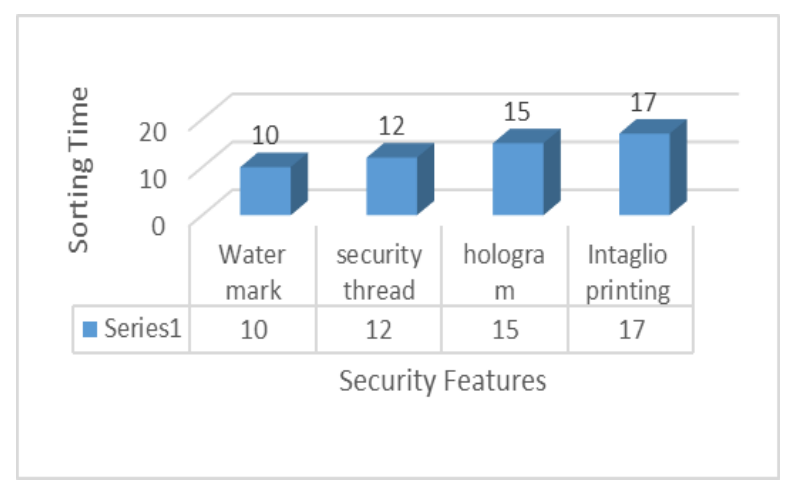

Figure 9 shows the average sorting time in seconds to detect genuine less clean Egyptian currency notes (100, 50, 20,10 .) pounds for public people.

- The average accuracy to detect genuine clean Egyptian currency notes $(100,50,20,10)$ pounds for public people $98 \%$.

- The average accuracy to detect genuine less clean Egyptian currency notes $(100,50,20,10)$ pounds for public people $96 \%$.

- The average accuracy to detect worn clean Egyptian currency notes $(100,50,20,10)$ pounds for public people $94 \%$.

- The average accuracy to detect worn less clean Egyptian currency notes $(100,50,20,10)$ pounds for public people $92 \%$.

- The average accuracy to detect counterfeiting clean Egyptian currency notes $(100,50,20,10)$ pounds for public people $96 \%$.

- The average accuracy to detect counterfeiting less clean Egyptian currency notes $(100,50,20,10)$ pounds for public people $94 \%$. 
- The cleanliness of banknotes circulation has an effect on the public's ability to distinguish between genuine banknotes and counterfeits. Counterfeits are easier to detect in a clean banknote circulation than in a less clean banknote circulation.

- In a clean banknote circulation, respondents are more suspicious and tend to declare more banknotes to be counterfeits.

- The average hit rate increasing with increase the number of security features checked.

- The average False alarm Rate decreased with increase the number of security features checked and this indicate the system performed well, being able to completely discriminate between the genuine and counterfeit banknotes.

- $\quad$ The average sorting time decreased with the cleanliness of banknotes and increased with less clean.

- The most important security features Egyptian currency notes $(100,50,20,10)$ acceptable for public people are Watermarked then security thread then hologram and finally Intaglio printing.

\section{VALIDATION}

Validation is the degree to which an assessment measures what it is supposed to measure. Essentially, there are three types of validity i) content validity, ii) criterion- related validity, and iii) construct validity. A questionnaire undergoes a validation procedure to make sure that it accurately measures what it aims to do, regardless of the responder. Valid questionnaire helps to collect better quality data with high comparability which reduces the effort and increase the credibility of data. A valid questionnaires must have following characteristics (i) simplicity and viability (ii) reliability and precision in the words (iii) adequate for the problem intended to measure (iv) reflect underlying theory or concept to be measured and (v) capable of measuring change.

\section{CONCLUSION}

This paper aimed to propose an intelligent fuzzy based framework for evaluating the most important security features of banknotes. A conceptual framework for user feedback analysis that provides a reference point for the community of stakeholders to consider how qualitative and quantitative feedback can help in making informed decisions with respect to evaluate the banknote as genuine, fake and worn. The results showed that counterfeits are easier to detect in a clean banknote circulation than in a less clean banknote circulation and the average sorting time decreased with the cleanliness of banknotes and increased with less clean. The average hit rate increasing with increase the number of security features checked. The average False alarm Rate decreased with increase the number of security features checked and this indicate the system performed well, being able to completely discriminate between the genuine and counterfeit banknotes. In this work, we investigated the most important security features Egyptian currency notes acceptable for public people are Watermarked then security thread then hologram and finally Intaglio printing. The proposed framework has several advantages it is general application, meaning that it can be applied for any banknote. It is simple meaning that can be used to code the banknotes straightforward and fast; most of them can be executed in fractions of a second on commercially available equipment.

\section{FUTURE WORK}

The ideas that are expected to be focused in the future include:

- Enhancing the framework by increase the data, number of the user and various Egyptian and foreign currencies in order to get bigger feedback.

- Enhancing the framework using the results of the application.

- Utilization of fuzzy neural network to enhance the proposed framework

- More security features of Egyptian banknotes may be detected.

\section{REFERENCES}

[1] Trond, E., and Leif, V., "Quality of banknotes in circulation - Norges Bank's role and monitoring system", Norges Bank, Staff Memo, NO. 7, 2016, pp. 1 17.

[2] Berenguel, A. Terrades, O. Liados, J. and, Cristina, C. "Banknotes counterfeit detection through background texture printing analysis", IEEE, Workshop on Document Analysis Systems, 2016.

[3] Mohammad, S. Pronaya, P. and, Shamim, A. "ImageBased Approach for the Detection of Counterfeit Banknotes of Bangladesh", 2016 5th International Conference on Informatics, Electronics and Vision (ICIEV), 13-14 May 2016, PP. 1067-1072.

[4] Marcela, M. and Richard, G. "Currency Design in the United States and Abroad: Counterfeit Deterrence and Visual Accessibility", Federal Reserve Bank of St. Louis Review, September/October 2007, 89(5), pp. 371-414.

[5] Swapna, G. Venky, S. and Sandy, G. "A Conceptual Framework for Analyzing Students' Feedback", Proceedings of 47th Annual Frontiers in Education Conference, Indianapolis, Indiana, USA, 2017 October 18-21.

[6] Qing, L. "A novel Likert scale based on fuzzy sets theory", Expert Systems with Applications, Expert Systems with Applications 40 (2013) 1609-1618.

[7] Atef, T. Nagy R., and Hesham, A. "Towards a Fuzzy based Framework for Effort Estimation in Agile Software Development", International Journal of Computer Science and Information Security, Vol. 13, No. 1, 2015, pp. 37-45.

[8] Harald, D. and Anna, L. "Modelling euro banknote quality in circulation, No 204 / December 2017.

[9] Cowling, A. and Monica, L. "Banknote Quality in Australia” June Quarter 2012.

[10] Frank, V. Martina, E. Susann, S. and Jelle, M. "Does banknote quality affect counterfeit detection? Experimental evidence from Germany and the Netherlands", Bundesbank Discussion paper NO. 06/2016.

[11] Rush, A. "The Life of Australian Banknotes", Note Issue Department Reserve Bank of Australia, August 2015.

[12] J. Geusebroek, and P. Markus"Learning Banknote Fitness for Sorting”, De Neder landsche Bank N.V. 
[13] Kwon, S. Pham, T. Park, K. Jeong, D. and, Yoon, S. "Recognition of Banknote Fitness Based on a Fuzzy System Using Visible Light Reflection and Near-infrared Light Transmission Images", Sensors 2016, 16, 863, pp. $1-18$.

[14] Andersen, M. "The Note Quality Reward Scheme" the Australian Banknote Distribution System, 16 Billetaria NO. 3 March 2008.

[15] Masuda, O. Pedersen, M. and Hardeberg, J. "Effects of awareness to security features on the confidence in banknotes", J. Print Media Technol. Res. 4(2015)2, 103110 .

[16] Masuda, O. Pedersen, M. and Hardeberg, J. "Features contributing to the genuineness of portraits on Nakamura, C. ,"The Security Printing Practices of banknotes" J. Print Media Technol. Res. 5(2016)1, 5359.
[17] Nakamura, C. "The Security Printing Practices of Banknotes", Project Faculty of the Graphic Communication California Polytechnic State University, San Luis Obispo.

[18] Hans, A."The design methodology of Dutch banknotes", International Symposium Electronic Imaging, California, USA, January, 2000.

[19] Hans, A. "Public feedback for better banknote design", Annual Symposium Electronic Imaging Conference, California, USA, January, 2006.

[20] Darwish, N. and, Abdelghany, A. "A Fuzzy Logic Model for Credit Risk Rating of Egyptian Commercial Banks", International Journal of Computer Science and Information Security, Vol. 14, No. 2, February 2016. 